\title{
Improved Screw Theory using Second Order Terms
}

\author{
Jun Takamatsu Hiroshi Kimura $\dagger$ Katsushi Ikeuchi \\ Institute of Industrial Science
}

\begin{abstract}
The local displacement of an object is very useful for deciding grasp stability, generating trajectories, recognizing assembly tasks, and so on. To calculate this displacement, the screw theory is employed. It is equivalent to the first order Taylor expansion of the displacement. The screw theory is very convenient, because the displacement is formulated as simultaneous linear inequalities, and a powerful tool to calculate such inequalities, the theory of the polyhedral convex cones, has already been established.

However, truncation errors introduced by first order approximations sometimes cause mistaken results. In this paper, we improve the screw theory by using 2nd order terms and verify the validity of the result.
\end{abstract}

\section{Introduction}

The local displacement of an object is very useful for deciding grasp stability[1, 2], generating trajectories [3, 4], recognizing assembly tasks[5, 6], and so on. The screw theory or tools with equivalent capabilities are employed for calculating the displacement. They are the first order Taylor expansion of the displacement. Therefore, the displacement is being formulated as simultaneous linear inequalities. That is a good characteristic because a powerful tool to calculate such inequalities, the theory of the polyhedral convex cones[7], has already been established.

However, truncation errors introduced by first order approximations sometimes cause mistaken results. For example, consider the case shown in Figure 1. We can easily see that the contact relation cannot be maintained if the white object rotates about the $y$ axis, which is perpendicular to this paper. However, we cannot recognize this with the screw theory. To overcome this problem, this paper considers the use of high order terms, especially second order terms.

Rimon and Burdick proposed an index for deciding grasp stability that employs second order terms[1]. However, the index can be employed only for this purpose. Research to employ high order terms does not, to our knowledge, exist. Therefore we propose

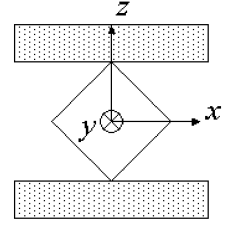

Figure 1: Case causing mistaken result
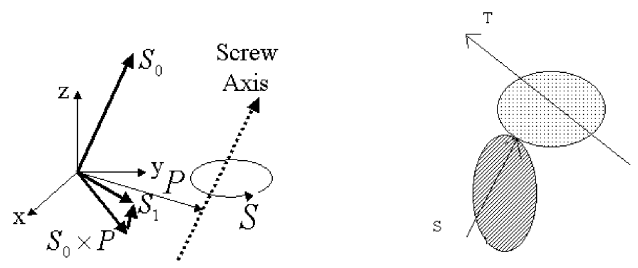

Figure 2: Screw repre-Figure 3: one object sentation is in contact with another

an improved screw theory for various types of applications, that also employs second order terms.

The contents of this paper are as follows: Section 2 illustrates the original screw theory. Sections 3 and 4 introduce an improved screw theory from local displacement. Section 5 applies it to a contact relation in which the original screw theory produces a mistaken result. Section 6 concludes this paper.

\section{Screw theory}

To represent infinitesimal displacement, we employ the screw representation[8]. It is represented as the combination of a translation along the screw axis and a rotation about the same axis (Shown in Figure 2). We can formulate the infinitesimal displacement by using the screw theory.

The screw is a convenient concept for representing three dimensional rigid body displacement. Any rigid body displacement can be accomplished by a rotation about a unique axis and a translation along 
the same axis. The combined motion is called a screw displacement or twist. The axis is referred to as the screw axis, and the ratio of the translation to the rotation is designated as the pitch of the screw. The amount of rotation about the screw axis is called the amplitude of the screw.

A screw $\mathbf{S}$ is represented mathematically by two 3D vectors, $\left[\mathbf{S}_{\mathbf{0}}, \mathbf{S}_{\mathbf{1}}\right] . \mathbf{S}_{\mathbf{0}}$ is the direction of the screw axis and $\mathbf{S}_{\mathbf{1}}=\mathbf{P} \times \mathbf{S}_{\mathbf{0}}+p \mathbf{S}_{\mathbf{0}}$, where the vector $\mathbf{P}$ is one from the origin to the screw axis and the scalar $p$ is the pitch of the screw. For pure rotation, the pitch $p$ is equal to 0 , and the screw $\mathbf{S}$ will be $\left[\mathbf{S}_{\mathbf{0}}, \mathbf{P} \times \mathbf{S}_{\mathbf{0}}\right]$. For pure translation, the pitch $p$ is infinity, therefore the screw $\mathbf{S}$ will be $\left[\mathbf{0}, \mathbf{S}_{\mathbf{0}}\right]$.

Consider two bodies in contact as shown in Figure 3. Let screw $\mathbf{S}=\left[\mathbf{S}_{\mathbf{0}}, \mathbf{S}_{\mathbf{1}}\right]$ represent the line of contact in $3 \mathrm{D}$ space and screw $\mathbf{T}=\left[\mathbf{T}_{\mathbf{0}}, \mathbf{T}_{\mathbf{1}}\right]$ represent the displacement of one object with respect to the other. Any screw $\mathbf{T}$ that can cause the sliding of object $\mathrm{B}$ on object A is called a reciprocal screw and is related to $\mathbf{S}$ by equation (1).

$$
\mathbf{S}_{\mathbf{0}} \cdot \mathbf{T}_{\mathbf{1}}+\mathbf{S}_{\mathbf{1}} \cdot \mathbf{T}_{\mathbf{0}}=0
$$

Any screw $\mathbf{T}$ that can cause the detaching of object $\mathrm{B}$ from object $\mathrm{A}$ is called a repelling screw and is related to $\mathrm{S}$ by equation (2).

$$
\mathbf{S}_{\mathbf{0}} \cdot \mathbf{T}_{\mathbf{1}}+\mathbf{S}_{\mathbf{1}} \cdot \mathbf{T}_{\mathbf{0}}>0
$$

Thus the relation that defines the feasible set of legal motions which do not violate the contact(sliding and repelling) can be written as equation (3).

$$
\mathbf{S}_{0} \cdot \mathbf{T}_{1}+\mathbf{S}_{1} \cdot \mathbf{T}_{\mathbf{0}} \geq 0
$$

\section{Improved screw theory}

We improve the screw theory as follows: First, we employ second order terms. Next, a center of rotation is variant to an amount of rotation, however an axis direction of rotation is invariant to the amount, because local displacement usually appearing has this characteristic.

Due to limitations of space, we are concerned here with only polyhedral objects. We believe that our proposed method is useful for objects with second order curved surfaces. However, it should not be useful for objects with more than second order curved surfaces, because of second order approximations of the local displacement.

Every contact relation between two polyhedral objects can be represented as a combination of vertexface $(\mathrm{V}-\mathrm{F})$, face-vertex $(\mathrm{F}-\mathrm{V})$, and non-parallel edgeedge(E-E) contacts as shown in Figure 4. And local displacement is usually formulated as simultaneous
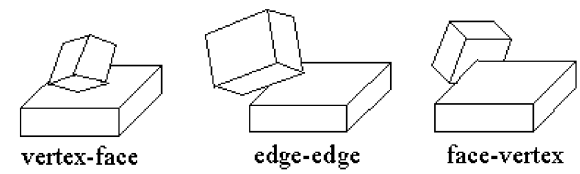

Figure 4: Essential three contacts

inequalities each representing a contact in the combination.

First, we formulate the local displacement in three types of contact relations. Next, we apply a second order Taylor expansion to them. We only concentrate on rotation. We consider translation after formulating the displacement in rotation.

\section{$3.1 \quad$ V-F contact}

Consider the case of a V-F contact as shown in Figure 5. The feasible displacement is formulated by equation (4), where $\mathbf{n}$ is a unit face normal vector, $\mathbf{v}$ is a vector from origin to the vertex, $\mathbf{r}=\left(r_{x}, r_{y}, r_{z}\right)$ is a right-screw axis direction of rotation, $\mathbf{c}$ is a center of rotation, $\Delta \theta$ is an amount of rotation, and $I$ is a $3 \times 3$ unit matrix.

$$
\begin{gathered}
\Delta_{v f}=\mathbf{n} \cdot(R(\mathbf{v}-\mathbf{c})+\mathbf{c}+s \mathbf{r}-\mathbf{v}) \geq 0 \\
R=I+\sin \Delta \theta[\mathbf{r}]_{\times}+(1-\cos \Delta \theta)[\mathbf{r}]_{\times}^{2} \\
{[\mathbf{r}]_{\times}=\left(\begin{array}{ccc}
0 & -r_{z} & r_{y} \\
r_{z} & 0 & -r_{x} \\
-r_{y} & r_{x} & 0
\end{array}\right)}
\end{gathered}
$$

Equation (5) is obtained by applying the Taylor expansion to equation (4) near $\Delta \theta=0$, where $\mathbf{t}_{\mathbf{1}}=$ $\mathbf{r}(\neq \mathbf{0}), \mathbf{t}_{\mathbf{2}}=\mathbf{c} \times \mathbf{r}+s_{1} \mathbf{r}, \mathbf{t}_{\mathbf{3}}=2 \mathbf{c}^{\prime} \times \mathbf{r}+s_{2} \mathbf{r}$. The sign $'$ means primary differentiation to $\Delta \theta$.

$$
\begin{aligned}
\Delta_{v f} & =\left((\mathbf{v} \times \mathbf{n}) \cdot \mathbf{t}_{\mathbf{1}}+\mathbf{n} \cdot \mathbf{t}_{\mathbf{2}}\right) \Delta \theta \\
& +\left(\mathbf{n} \cdot \mathbf{t}_{\mathbf{3}}+\left(\mathbf{n} \times \mathbf{t}_{\mathbf{1}}\right) \cdot\left(\mathbf{t}_{\mathbf{1}} \times \mathbf{v}+\mathbf{t}_{\mathbf{2}}\right)\right) \frac{\Delta \theta^{2}}{2} \\
& +O\left(\Delta \theta^{3}\right)
\end{aligned}
$$

Naturally the coefficient of $\Delta \theta$ is equivalent to the original screw theory.

\subsection{F-V contact}

Consider the case of an F-V contact as shown in Figure 6 . The feasible displacement is formulated by equation (6).

$$
\Delta_{f v}=(R \mathbf{n}) \cdot(\mathbf{v}-R(\mathbf{v}-\mathbf{c})-\mathbf{c}-s \mathbf{r}) \geq 0
$$

Equation (7) is obtained by applying the Taylor expansion to equation (6) near $\Delta \theta=0$. Naturally, it is 


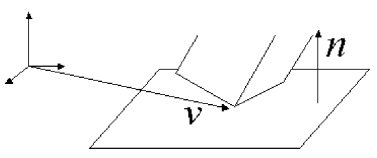

Figure 5: V-F contact

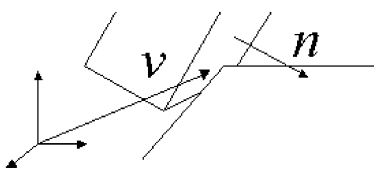

Figure 6: $F-V$ contact

the same as when substituting $\mathbf{t}_{\mathbf{1}}=-\mathbf{t}_{\mathbf{1}}, \mathbf{t}_{\mathbf{2}}=-\mathbf{t}_{\mathbf{2}}$, and $\mathbf{t}_{\mathbf{3}}=-\mathbf{t}_{\mathbf{3}}$ in equation (5).

$$
\begin{aligned}
\Delta_{f v} & =-\left((\mathbf{v} \times \mathbf{n}) \cdot \mathbf{t}_{\mathbf{1}}+\mathbf{n} \cdot \mathbf{t}_{\mathbf{2}}\right) \Delta \theta \\
& +\left(-\mathbf{n} \cdot \mathbf{t}_{\mathbf{3}}+\left(\mathbf{n} \times \mathbf{t}_{\mathbf{1}}\right) \cdot\left(\mathbf{t}_{\mathbf{1}} \times \mathbf{v}+\mathbf{t}_{\mathbf{2}}\right)\right) \frac{\Delta \theta^{2}}{2} \\
& +O\left(\Delta \theta^{3}\right)
\end{aligned}
$$

\subsection{E-E contact}

Consider the case of an E-E contact as shown in Figure 7 . The feasible displacement is formulated by equation (8).

$$
\Delta_{e e}=\frac{\left(\mathbf{R l}_{\mathbf{1}} \times \mathbf{l}_{\mathbf{2}}\right) \cdot(\mathbf{R}(\mathbf{v}-\mathbf{c})+\mathbf{c}+s \mathbf{r}-\mathbf{v})}{\left|\mathbf{R l}_{\mathbf{1}} \times \mathbf{l}_{\mathbf{2}}\right|} \geq 0
$$

We need only to focus on the numerator for the displacement. Equation (9) is obtained by applying the Taylor expansion to the numerator of equation (8) near $\Delta \theta=0$, where $\mathbf{n}=\mathbf{l}_{\mathbf{1}} \times \mathbf{l}_{\mathbf{2}}$. However, the directions of $\mathbf{l}_{\mathbf{1}}, \mathbf{l}_{\mathbf{2}}$ must be selected as the direction of $\mathbf{n}$ outside of an object.

$$
\begin{aligned}
\Delta_{e e}= & \left((\mathbf{v} \times \mathbf{n}) \cdot \mathbf{t}_{\mathbf{1}}+\mathbf{n} \cdot \mathbf{t}_{\mathbf{2}}\right) \Delta \theta \\
+ & \left(\mathbf{n} \cdot \mathbf{t}_{\mathbf{3}}+\left(\mathbf{n} \times \mathbf{t}_{\mathbf{1}}\right) \cdot\left(\mathbf{t}_{\mathbf{1}} \times \mathbf{v}+\mathbf{t}_{\mathbf{2}}\right)\right. \\
& \left.+2\left(\left(\mathbf{t}_{\mathbf{1}} \times \mathbf{l}_{\mathbf{1}}\right) \times \mathbf{l}_{\mathbf{2}}\right) \cdot\left(\mathbf{t}_{\mathbf{1}} \times \mathbf{v}+\mathbf{t}_{\mathbf{2}}\right)\right) \frac{\Delta \theta^{2}}{2} \\
+ & O\left(\theta^{3}\right)
\end{aligned}
$$

\subsection{Translation}

We formulated local displacement in rotation. For translation, the equation can easily be formulated as

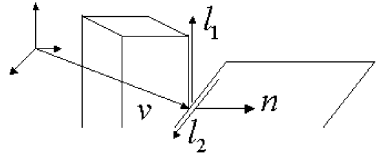

Figure 7: E-E contact

$\mathbf{t}_{\mathbf{1}}=\mathbf{0}$. This can be seen by substituting $\mathbf{t}_{\mathbf{1}}=\mathbf{0}$ in equations (5), (7), and (9). The factor $\mathbf{t}_{\mathbf{1}}=\mathbf{0}$ representing translation corresponds to a screw vector $\left[\mathbf{0}, \mathbf{T}_{\mathbf{1}}\right]$ representing pure translation. Therefore, we need not to classify translation and rotation when we calculate local displacement.

\section{Solving local displacement}

Before beginning to describe the method to solve local displacement, we should point out that $\mathbf{t}_{\mathbf{1}}$, $\mathbf{t}_{\mathbf{2}}$, and $\mathbf{t}_{\mathbf{3}}$ can be set to any values independently. Therefore, we can treat these variables independently.

From the formulation we can see that, when the coefficient of $\Delta \theta$ is greater than zero, the displacement corresponding to $\Delta \theta>0$ is repelling motion, and when the coefficient is less than zero, the displacement is illegal. When the coefficient is equal to zero, the displacement, however, depends on a coefficient of $\Delta \theta^{2}$, therefore, it is not always sliding motion.

The problem of an improved screw theory is to classify first order sliding motion into three types; second order repelling, illegal, or sliding motion. In this paper, we focus on classifying first order sliding motion into two types; second order sliding motion or not.

Let equation (10) represent the feasible displacement of the i-th contact. Naturally equation (10) is equal to equation $(5),(7)$, or (9).

$$
\Delta=f_{i}\left(\mathbf{t}_{\mathbf{1}}, \mathbf{t}_{\mathbf{2}}\right) \Delta \theta+g_{i}\left(\mathbf{t}_{\mathbf{1}}, \mathbf{t}_{\mathbf{2}}, \mathbf{t}_{\mathbf{3}}\right) \Delta \theta^{2} \geq 0
$$

Second order sliding motion must satisfy equation (11).

$$
\forall i\left(f_{i}=0 \cap g_{i}=0\right)
$$

The condition, $\forall i\left(f_{i}=0\right)$, is a system of simultaneous linear equalities, therefore, we can solve them as equation (12), where $\mathbf{b}_{\mathbf{i}}$ is an $\mathbf{i}$-th six-dimensional basis.

$$
\left[\mathbf{t}_{\mathbf{1}}, \mathbf{t}_{\mathbf{2}}\right]=\sum_{i=1}^{r} s_{i} \mathbf{b}_{\mathbf{i}}
$$

Next, equation (13) is obtained by substituting equation (12) in $g_{i}$.

$$
g_{i}=\mathbf{n}_{\mathbf{i}} \cdot \mathbf{t}_{\mathbf{3}}+h_{i}\left(s_{1}, \ldots, s_{r}\right)
$$


Because $g_{i}$ is a coefficient of $\Delta \theta^{2}$ in the equations (5), $(7)$, or $(9), h_{i}$ must be a second order homogeneous polynomial.

To solve the condition $\forall i\left(g_{i}=0\right)$, first we search all linear dependent combinations, $C_{j}$, of $\left\{n_{i}\right\}$, and acquire equations as shown in equation (14), where $a_{i}$ is any real number.

$$
k_{i}=\sum_{j \in C_{i}} a_{j} h_{j}=0
$$

All spaces $\left(s_{1}, \cdots, s_{r}\right)$ represent first order sliding motion. Among those, sub-spaces to be restrained by equation (14) represent second order not-sliding motion, and other sub-spaces represent second order sliding motion.

Because the equation $k_{i}$ also must be a second order homogeneous polynomial, it can be represented by equation (15), where $K_{i}$ is a symmetric matrix and $\mathbf{s}^{T}=\left(s_{1} \cdots s_{r}\right)$.

$$
k_{i}=\mathbf{s}^{T} K_{i} \mathbf{s}
$$

Then a matrix $K_{i}$ can be represented by $A^{T} W A$, where $A$ is an orthogonal matrix and $W$ is a diagonal matrix, because $K$ is a symmetric matrix.

$$
k_{i}=(A \mathbf{s})^{T} W A \mathbf{s}=0
$$

Equation (16) can be decomposed as linear equations as shown in equation (17), if the signs of all diagonal elements, $w_{i}$, of $W$ are the same, because each $s_{i}$ is a real number.

$$
\bigcap_{i \in\left(w_{i} \neq 0\right)} \sum_{j} a_{i j} s_{j}=0
$$

Equation (16) can be decomposed in linear equations as shown in equation (18), if $\exists i, j\left(w_{i}>0 \cap w_{j}<\right.$ $\left.0 \cap \forall(k \neq i, j)\left(w_{k}=0\right)\right)$.

$$
\bigcup_{i \in\left(w_{i} \neq 0\right)} \sum_{j} a_{i j} s_{j}=0
$$

To substitute such linear equations obtained by the above mentioned method may enable all second order homogeneous equations to be decomposed. We believe that usually all the equations can be decomposed because local displacement may be usually represented as linear equations.

In some special cases, all equations may not be decomposed. To overcome this problem, we may be able to employ the concept of the Groebner basis. However, a second order polynomial is not convenient to treat. We think our proposed method is very easy and very useful, because it treats only linear equations and is superior to the original screw theory.
Finally, we prove that the result obtained by the above mentioned method is correct. For that, we prove two things as follows: one is that the second order sliding motion obtained is correct, and the other is that the second order non-sliding motion obtained is correct. The latter thing is obvious. So we prove the former thing.

Proposition 1 The second order sliding motion obtained by the method mentioned above is correct, that is, all regions $\left(s_{1}, \cdots s_{r}\right)$ not to be restrained by the equations (14) satisfy the equation (11).

Proof Now, we assume that a region not satisfying the equation (11) exists. Then, $h_{i}$ is a constant real number. Because the equation, $\forall i\left(\cap g_{i}=0\right)$, does not have a solution, a combination, $\left\{j_{1}, \ldots j_{m}\right\}$, must exist, where the rank of a matrix $A=m-1$, the rank of a matrix $[A \mathbf{b}]=m$, and

$$
A=\left(\begin{array}{l}
n_{j_{1}}^{T} \\
\vdots \\
n_{j_{m}}^{T}
\end{array}\right), \mathbf{b}=\left(\begin{array}{l}
h_{j_{1}} \\
\vdots \\
h_{j_{m}}
\end{array}\right) .
$$

Because the rank of a matrix $A=m-1$, $\left\{n_{j_{1}}, \ldots n_{j_{m}}\right\}$ is linear dependent. Because the rank of a matrix $A$ is different from the rank of a matrix $[A \mathbf{b}]$, the equation (14) obtained by the combination must not be zero. That is a contradiction.

\section{Example}

The original screw theory usually produces correct results. Because our proposed method is correct when the original screw theory is, we apply the method to the case in which the original screw theory produces mistaken results.

The contact relation as shown in Figure 1 is adequate to verify the validity of the method. In this case, four F-V contacts exist. Locations of all of the vertices are $(0,20,10),(0,20,-10),(0,-20,10)$, and $(0,-20,-10)$.

First, we formulate feasible first order displacement as equation (19).

$$
\left(\begin{array}{cccccc}
10 & 0 & 0 & 0 & 0 & -1 \\
-10 & 0 & 0 & 0 & 0 & 1 \\
-10 & 0 & 0 & 0 & 0 & -1 \\
10 & 0 & 0 & 0 & 0 & 1
\end{array}\right)\left(\begin{array}{l}
\mathbf{t}_{1} \\
\mathbf{t}_{\mathbf{2}}
\end{array}\right) \geq\left(\begin{array}{l}
0 \\
0 \\
0 \\
0
\end{array}\right)
$$

We can solve equation (19) as equation (20), that means an object cannot translate along the z-axis and rotate about the $\mathrm{x}$-axis, and other displacements are first order slide motions.

$$
\left(\begin{array}{cccccc}
10 & 0 & 0 & 0 & 0 & -1 \\
-10 & 0 & 0 & 0 & 0 & -1
\end{array}\right)\left(\begin{array}{l}
\mathbf{t}_{\mathbf{1}} \\
\mathbf{t}_{\mathbf{2}}
\end{array}\right)=\left(\begin{array}{l}
0 \\
0
\end{array}\right)
$$


We classify all first order slide motion into second order slide motion or not. We select the basis of first order slide motion as equation (21)

$$
\begin{aligned}
& \mathbf{s}_{\mathbf{1}}=(0,1,0,0,0,0) \\
& \mathbf{s}_{\mathbf{2}}=(0,0,0,1,0,0) \\
& \mathbf{s}_{\mathbf{3}}=(0,0,0,0,1,0) \\
& \mathbf{s}_{\mathbf{4}}=(0,0,-1,0,0,0)
\end{aligned}
$$

Next, we obtain equation (22) which second order slide motion must satisfy.

$$
\begin{aligned}
(0,0,-1) \cdot \mathbf{t}_{\mathbf{3}}+20 s_{1}^{2}+2 s_{1} s_{2}-20 s_{1} s_{4} & =0 \\
(0,0,1) \cdot \mathbf{t}_{\mathbf{3}}+20 s_{1}^{2}-2 s_{1} s_{2}+20 s_{1} s_{4} & =0 \\
(0,0,-1) \cdot \mathbf{t}_{3}+20 s_{1}^{2}+2 s_{1} s_{2}+20 s_{1} s_{4} & =0 \\
(0,0,1) \cdot \mathbf{t}_{3}+20 s_{1}^{2}-2 s_{1} s_{2}-20 s_{1} s_{4} & =0(22)
\end{aligned}
$$

We obtain equation (23) by searching linear dependent combinations.

$$
\begin{aligned}
40 s_{1}^{2} & =0 \\
-40 s_{1} s_{4} & =0 \\
40 s_{1}^{2}-40 s_{1} s_{4} & =0 \\
40 s_{1}^{2}+40 s_{1} s_{4} & =0 \\
40 s_{1} s_{4} & =0 \\
40 s_{1}^{2} & =0
\end{aligned}
$$

We obtain $s_{1}=0$ by solving equation (23). This means rotation about the y-axis cannot maintain the contact relation, i.e. is not a second order slide motion. But other first order slide motions are also second order slide motions. The result is obviously correct.

\section{Conclusion}

We proposed a method to improve the screw theory using second order terms. First, we illustrated a problem of the original screw theory and described the use of second order terms to overcome this problem. Next, we introduced the improved screw theory by applying second order Taylor expansion to the local displacement of an object. Then, we proposed a method to solve the resulting equations. Next, we verified the validity of our proposed method by applying it to the case where the original screw theory produced a mistaken result.

Due to space limitations, we could not illustrate the method for objects with second order curved surfaces. However, we examined that our method was probably useful for them through various trials.

This paper proposed a method to classify first order slide motion into second order slide motion or not. However, we must introduce a method to classify it into three types; repelling, illegal or slide motion. That is an open problem.

\section{Acknowledgments}

This work is supported in part by the Japan Science and Technology Corporation (JST) under the Ikeuchi CREST project, and in part by the Grant-in-Aid for Scientific Research on Priority Areas (C) 13224025 of the Ministry of Education, Culture, Sports, Science and Technology.

\section{References}

[1] E. Rimon and J. Burdick : "Mobility of bodies in contact - i: A new 2nd order mobility index for multiple-finger grasps," IEEE Inter. Conf. on Robotics and Automation, pp. 2329 - 2355, 1994.

[2] S. Hirai : "Kinematics and statics of manipulation using the theory of polyhedral convex cones and their application to the planning of manipulative operations," Journal of the Robotics Society of Japan, Vol. 17, No. 1, pp. 68 -83, 1999.

[3] H. Hirukawa, T. Matsui, and K. Takase : "A fast algorithm for the analysis of the constraint for motion of polyhedra in contact and its application to departure motion planning," Journal of the Robotics Society of Japan, Vol. 9, No. 7, pp. $841-848,1991$.

[4] X. Ji and J. Xiao : "Automatic generation of highlevel contact state space," Proc. of Inter. Conf. on Robotics and Automation, pp. 238 - 244, 1999.

[5] K. Ikeuchi and T. Suehiro : "Toward an assembly plan from observation part i: Task recognition with polyhedral objects," IEEE Trans. on Robotics and Automation, Vol. 10, No. 3, 1994.

[6] J. Takamatsu, H. Tominaga, K. Ogawara, H. Kimura, and K. Ikeuchi : "Extracting manipulation skills from observation," IEEE Inter. Conf. on Intelligent Robots and Systems, Vol. 1, pp. 584 $-589,2000$.

[7] H. W. Kuhn and A. W. Tucker : "Linear inequalities and related systems," Annals. of Mathematics Studies, Vol. 38, , 1956.

[8] M. S. Ohwovoriole and B. Roth : "An extension of screw theory," Journal of Mechanical Design, Vol. 103, pp. 725 - 735, Oct. 1981. 